Preprint typeset in JHEP style. - HYPER VERSION

\title{
Soliton-preserving boundary condition in affine Toda field theories
}

\author{
Gustav W Delius \\ Department of Mathematics, King's College London, \\ Strand, London WC2R 2LS, UK. \\ E-mail: delius@mth.kcl.ac.uk \\ $W W W:$ http://wWW.mth.kcl.ac.uk/ delius/
}

\begin{abstract}
We give a new integrable boundary condition in affine Toda theory which is soliton-preserving in the sense that a soliton hitting the boundary is reflected as a soliton. All previously known integrable boundary conditions forced a soliton to be converted into an antisoliton upon reflection. We prove integrability of our boundary condition using a generalization of Sklyanin's formalism.
\end{abstract}




\section{Introduction}

Associated to every complex affine Lie algebra $\hat{g}$ there is an integrable relativistic field theory in $1+1$ dimensions, called affine Toda theory, described by the equations of motion

$$
\ddot{\boldsymbol{\phi}}-\boldsymbol{\phi}^{\prime \prime}+m^{2} \sum_{i=0}^{n} \eta_{i} \boldsymbol{\alpha}_{i} e^{\boldsymbol{\alpha}_{i} \cdot \boldsymbol{\phi}}=0 .
$$

Here $\boldsymbol{\phi}$ is an n-component bosonic field, where $n$ is the rank of $\hat{g} . \boldsymbol{\alpha}_{i}, i=1, \ldots, n$ are the simple roots of the finite dimensional Lie algebra $g$ underlying $\hat{g}$ and $\boldsymbol{\alpha}_{0}=$ $-\sum_{i=1}^{n} \eta_{i} \boldsymbol{\alpha}_{i}$ is the extra simple root that needs to be added to obtain the extended Dynkin diagram of $\hat{g}$. We have defined $\eta_{0}=1 . m$ is a mass parameter and we will choose units so that $c=m=1$ throughout this letter. We have rescaled the fields so that the coupling constant $\beta$ of affine Toda theory does not enter the equations of motion. The simplest affine Toda theory is that for $g=\operatorname{sl}(2)$ which is the sinhGordon theory (or the sine-Gordon theory after rescaling the field by a factor of $i$ ). For a review of affine Toda theory see for example [5].

The integrability of affine Toda theories manifests itself in the existence of soliton solutions [13, 18]. These are kink configurations interpolating between different minima of the potential. The potential corresponding to the equations of motion (1.1) is $V[\boldsymbol{\phi}]=\sum_{i=0}^{n} \eta_{i}\left(e^{\boldsymbol{\alpha}_{i} \cdot \boldsymbol{\phi}}-1\right)$ and its degenerate minima are at $\boldsymbol{\phi}=2 \pi i \boldsymbol{\lambda}$ where $\boldsymbol{\lambda}$ are the coweights of $g$. The special property of solitons is that when two of them meet they pass through each other and reemerge with their original shape. In the quantum theory the solitons give rise to particle states and their S-matrices have been determined [14, 10].

The first affine Toda theory to be studied in the presence of integrable boundaries was the sine-Gordon theory 12, 16, 19. The first necessary step to extend this to higher affine Toda theories is to

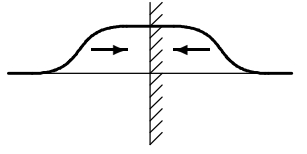

before

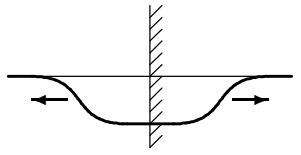

after find those boundary conditions which preserve integrability. This was done by the Durham group in [四, 6] by studying the

Figure 1: Soliton with mirror antisoliton satisfies Neumann condition (1.3). first higher-spin integrals of motion and then more systematically in [1] by constructing the generating functional for all higher-spin integrals of motion using a Lax pair construction. They find integrable boundary conditions of the form

$$
\left.\partial_{x} \boldsymbol{\phi}\right|_{\text {boundary }}=\left.\sum_{i=0}^{n} A_{i} \sqrt{2 \eta_{i} /\left|\boldsymbol{\alpha}_{i}\right|^{2}} \boldsymbol{\alpha}_{i} e^{\boldsymbol{\alpha}_{i} \cdot \boldsymbol{\phi} / 2}\right|_{\text {boundary }} .
$$

The coefficients $A_{i}$ specify the boundary condition. In the sine-Gordon model the $A_{i}$ are allowed to take arbitrary values but in all other affine Toda theories either all 
the $A_{i}$ must be zero (the Neumann boundary condition) or else most of the $A_{i}$ are restricted to be \pm 1 if integrability is to be preserved [1].

The existence of an infinite set of integrals of motion in involution is one hallmark of integrability, the existence of soliton solutions is another. In [7] we showed that it is indeed possible to construct soliton solutions satisfying boundary conditions of the form (1.2). We used a method of mirror images. Let us recall our method in the simplest case of the Neumann boundary condition $\partial_{x} \phi=0$ at $x=0$. Under parity reversal $\phi(x) \rightarrow \tilde{\phi}(x)=\phi(-x)$ a soliton with center of mass $X$ moving with velocity $v$ is transformed into an antisoliton with center of mass $-X$ and velocity $-v$. Therefore if $\phi$ describes a multisoliton configuration in which every soliton is paired with an oppositely moving antisoliton centered around the parity-transformed point, then $\phi$ is invariant under parity,

$$
\phi(-x)=\phi(x) \text { and thus } \partial_{x} \phi(x=0)=0 .
$$

Viewed on the left half line such a configuration describes solitons hitting the boundary and coming back as antisolitons. This is described schematically in figure 1 .

In this letter we want to find a boundary condition which ensures that solitons come back as solitons after reflection. Thus we want a condition which selects those multisoliton solutions on the whole line in which each soliton is paired with a mirror soliton rather than

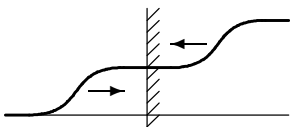

before

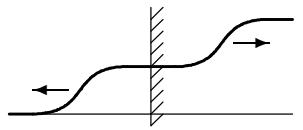

after

Figure 2: Soliton with mirror soliton satisfies the new condition (1.4).

a mirror antisoliton, see figure 2. Using

the observation that complex conjugation transforms a soliton configuration into an antisoliton configuration we see that the appropriate modification of the condition (1.3) is

$$
\boldsymbol{\phi}^{*}(-x)=\phi(x)+2 \pi i \boldsymbol{\lambda} \text { with } \boldsymbol{\lambda} \in \text { coweight lattice of } g .
$$

The freedom is due to the fact that adding $2 \pi i$ times a coweight to a soliton solution gives an equivalent soliton solution. The boundary condition corresponding to (1.4) is

$$
\left.\frac{\operatorname{Im}(\phi)}{2 \pi}\right|_{\text {boundary }} \in \text { coweight lattice of } g \quad \text { and }\left.\quad \partial_{x} \operatorname{Re}(\phi)\right|_{\text {boundary }}=0 \text {, }
$$

It is a combination of a Dirichlet boundary condition for the imaginary part of the field and a Neumann boundary condition for the real part.

In the sine-Gordon model the field is purely imaginary (in our conventions). Thus in that case the boundary condition (1.5) is simply a Dirichlet condition which was known to be integrable. In general affine Toda theories one has to include 
complex field configurations in order to have solitons. The boundary condition (1.5) is the integrable generalization of the Dirichlet condition. Note that unlike in the sine-Gordon case there is no free parameter in the boundary condition (1.5).

It is intuitively clear that the new boundary condition (1.5) preserves exactly half of the infinitely many conserved charges of the theory on the whole line: when a right moving soliton is about to leave the left half line and to carry some charge away with it, a left moving soliton is moving onto the left half line. If a left moving soliton carries the same amount of charge as a right moving one, then the amount of charge on the left half line will be conserved. This is so exactly for half of all the charges. In the following sections we will go beyond this intuitive argument and construct a generating functional for the integrals of motion. We will use a generalization of Sklyanin's formalism [20], which we find conceptually slightly simpler than the approach of [1], and which works for intervals as well as the half line. We will also review the Durham boundary conditions in this formalism. The reason why the boundary condition (1.5) was not discovered in [1] is that there the field was always restricted to be purely real.

\section{Sklyanin's formalism for integrable field theories with bound- aries}

Sklyanin's original presentation of his method in his two page paper [20] is very succinct and clear. We present it here in a slightly generalized form needed for affine Toda theory. The method applies to spin chains as well as to field theories in two dimensions, we concentrate on the later.

It is assumed that there exists a pair of functions $a_{x}(\lambda)$ and $a_{t}(\lambda)$ which take their values in some Lie algebra $g$ and which depend on the fields of the theory, their conjugate momenta, and on an extra real parameter $\lambda$, so that the equations of motion of the field theory on the whole line are equivalent to the Lax pair equation

$$
\left[\partial_{x}+a_{x}(\lambda), \partial_{t}+a_{t}(\lambda)\right]=0 \quad \forall \lambda
$$

The utility of the extra parameter $\lambda$, called spectral parameter, will become apparent later. $a_{x}$ and $a_{t}$ depend on $x$ and $t$ only implicitly through their dependence on the fields. $\partial_{x}$ and $\partial_{t}$ denote total differentiation with respect to the space or time variable. A concrete example of a Lax pair will be given for affine Toda theory in eq.(3.1). If $a_{x}$ and $a_{t}$ are thought of as the components of a connection then eq.(2.1) is the zero curvature condition for this connection.

It is furthermore assumed that the canonical Poisson brackets for the fields give rise to Poisson brackets for the functions $a_{x}(\lambda ; x)$ of the form

$$
\left\{\stackrel{1}{a}_{x}\left(\lambda_{1} ; x\right), \stackrel{2}{a} x\left(\lambda_{2} ; y\right)\right\}=\delta(x-y)\left[r\left(\lambda_{1} / \lambda_{2}\right), \stackrel{1}{a}{ }_{x}\left(\lambda_{1} ; x\right)+\stackrel{2}{a}{ }_{x}\left(\lambda_{2} ; y\right)\right] .
$$


This is to be understood as an equation in $(g \oplus 1) \otimes(g \oplus 1)$. We used the shorthand notation $\stackrel{1}{a}_{x}=a_{x} \otimes 1$ and $\stackrel{2}{a}_{x}=1 \otimes a_{x} . r$ is an element of $g \otimes g$ which satisfies the classical Yang-Baxter equation. It is assumed to be constant and independent of the fields. In 20] Sklyanin requires that the $r$-matrix satisfies $r(\lambda)=-r(1 / \lambda)$. We drop this requirement because it is not essential and is not satisfied by the $r$-matrix (3.4) of affine Toda theory.

We now restrict the theory to an interval $\left[x_{-}, x_{+}\right]$. We define the "transfer matrix" $T(\lambda)$ as the path ordered exponentials of $a_{x}$ from $x_{-}$to $x_{+}$,

$$
T(\lambda)=P \exp \left(\int_{x_{-}}^{x_{+}} a_{x}(\lambda, x) d x\right) .
$$

The path ordered exponential is defined so that the operators at points nearer to $x_{+}$ are further to the left. These $T(\lambda)$ satisfy

$$
\begin{aligned}
\partial_{x_{+}} T(\lambda) & =a_{x}\left(\lambda ; x_{+}\right) T(\lambda), \\
\partial_{t} T(\lambda) & =a_{t}\left(\lambda ; x_{+}\right) T(\lambda)-T(\lambda) a_{t}\left(\lambda ; x_{-}\right) .
\end{aligned}
$$

The Poisson bracket relation (2.2) leads to

$$
\left\{\stackrel{1}{T}\left(\lambda_{1}\right), \stackrel{2}{T}\left(\lambda_{2}\right)\right\}=\left[r\left(\lambda_{1} / \lambda_{2}\right), \stackrel{1}{T}\left(\lambda_{1}\right) \stackrel{2}{T}\left(\lambda_{2}\right)\right] .
$$

In practice one will often work in a specific chosen representation of $g$. We will use the same symbols for Lie algebra valued objects and for the corresponding representation matrices.

Following Sklyanin one can prove the following proposition by straightforward calculation.

Proposition 1 Let the Lax connection $a_{\mu}(\lambda)$, the transfer matrix $T(\lambda)$ and the $r$ matrix $r(\lambda)$ be defined as above, but now written as matrices in some chosen representation. Let $\sigma$ be a linear or anti-linear involutive anti-automorphism of the Lie algebra g. Let $K_{ \pm}(\lambda)$ be two matrix valued functions of the spectral parameter $\lambda$, not depending on the fields or space-time, satisfying the classical reflection equations

$$
\begin{aligned}
& 0=\stackrel{1}{K}+\left(\lambda_{1}\right) \stackrel{2}{K}+\left(\lambda_{2}\right) r\left(\lambda_{1} / \lambda_{2}\right)+r^{\sigma_{1} \sigma_{2}}\left(\lambda_{2} / \lambda_{1}\right) \stackrel{1}{K}+\left(\lambda_{1}\right) \stackrel{2}{K}+\left(\lambda_{2}\right) \\
& +\stackrel{1}{K}+\left(\lambda_{1}\right) r^{\sigma_{2}}\left(\lambda_{1} \lambda_{2}\right) \stackrel{2}{K}+\left(\lambda_{2}\right)+\stackrel{2}{K}+\left(\lambda_{2}\right) r^{\sigma_{1}}\left(1 /\left(\lambda_{1} \lambda_{2}\right)\right) \stackrel{1}{K}{ }_{+}\left(\lambda_{1}\right), \\
& 0=\stackrel{1}{K}-\left(\lambda_{1}\right) \stackrel{2}{K}-\left(\lambda_{2}\right) r^{\sigma_{1} \sigma_{2}}\left(\lambda_{1} / \lambda_{2}\right)+r\left(\lambda_{2} / \lambda_{1}\right) \stackrel{1}{K}-\left(\lambda_{1}\right) \stackrel{2}{K}-\left(\lambda_{2}\right) \\
& +\stackrel{1}{K}-\left(\lambda_{1}\right) r^{\sigma_{1}}\left(\lambda_{1} \lambda_{2}\right) \stackrel{2}{K}-\left(\lambda_{2}\right)+\stackrel{2}{K}-\left(\lambda_{2}\right) r^{\sigma_{2}}\left(1 /\left(\lambda_{1} \lambda_{2}\right)\right) \stackrel{1}{K} K_{-}\left(\lambda_{1}\right),
\end{aligned}
$$

where by $r^{\sigma_{1}}$ we mean the result of acting with the involution $\sigma$ on the first factor of $r$ and similarly for $r^{\sigma_{2}}$ and $r^{\sigma_{1} \sigma_{2}}$. Then: 
a) The function

$$
\mathcal{T}(u)=T(\lambda) K_{-}(1 / \lambda) T^{\sigma}(1 / \lambda)
$$

obeys the Poisson bracket relation

$$
\begin{aligned}
\left\{\mathcal{T}_{\mathcal{T}}\left(\lambda_{1}\right), \stackrel{2}{\mathcal{T}}\left(\lambda_{2}\right)\right\} & =r\left(\lambda_{1} / \lambda_{2}\right) \stackrel{1}{\mathcal{T}}\left(\lambda_{1}\right) \stackrel{2}{\mathcal{T}}\left(\lambda_{2}\right)+\stackrel{1}{\mathcal{T}}\left(\lambda_{1}\right) \stackrel{2}{\mathcal{T}}\left(\lambda_{2}\right) r^{\sigma_{1} \sigma_{2}}\left(\lambda_{2} / \lambda_{1}\right) \\
& +\stackrel{2}{\mathcal{T}}\left(\lambda_{2}\right) r^{\sigma_{2}}\left(\lambda_{1} \lambda_{2}\right) \stackrel{1}{\mathcal{T}}\left(\lambda_{1}\right)+\stackrel{1}{\mathcal{T}}\left(\lambda_{1}\right) r^{\sigma_{1}}\left(1 /\left(\lambda_{1} \lambda_{2}\right)\right) \stackrel{2}{\mathcal{T}}\left(\lambda_{2}\right)
\end{aligned}
$$

b) The quantities

$$
\tau(\lambda)=\operatorname{tr}\left(K_{+}(\lambda) \mathcal{T}(\lambda)\right)=\operatorname{tr}\left(K_{+}(\lambda) T(\lambda) K_{-}(1 / \lambda) T^{\sigma}(1 / \lambda)\right)
$$

are in involution for any two values of the spectral parameter,

$$
\left\{\tau\left(\lambda_{1}\right), \tau\left(\lambda_{2}\right)\right\}=0, \quad \forall \lambda_{1}, \lambda_{2} \in \mathbb{R}
$$

c) If the the boundary conditions on the fields at $x_{-}$and $x_{+}$are such that they lead to

$$
\begin{aligned}
& K_{+}(\lambda) a_{t}\left(\lambda ; x_{+}\right)+a_{t}^{\sigma}\left(1 / \lambda ; x_{+}\right) K_{+}(\lambda)=0, \\
& K_{-}(\lambda) a_{t}^{\sigma}\left(\lambda ; x_{-}\right)+a_{t}\left(1 / \lambda ; x_{-}\right) K_{-}(\lambda)=0,
\end{aligned}
$$

then the quantities $\tau(\lambda)$ defined in eq. (2.11) are time conserved,

$$
\frac{d}{d t} \tau(\lambda)=0, \quad \forall \lambda \in \mathbb{R}
$$

One obtains Sklyanin's result [20] from this by choosing the anti-automorphism $\sigma$ to be the antipode ${ }^{1}$ and by assuming that $r(1 / \lambda)=-r(\lambda)$. Sklyanin [20] writes his formulas in terms of an additive spectral parameter $u$ rather than the multiplicative spectral parameter $\lambda=e^{u}$ which we use. The generalization of allowing antiautomorphisms other than the antipode was suggested to us by a similar generalization of the quantum reflection equation [15]. Something similar has also been found in [3].

The proposition implies that we can take $\tau$ as the generating functional for the integrals of motion in involution. If $\tau(\lambda)$ has a non-trivial dependence on $\lambda$ so that it generates an infinite number of independent integrals of motion, the field theory on the interval $\left[x_{-}, x_{+}\right]$with equations of motion given by (2.1) and boundary conditions given by (2.13) is integrable.

\footnotetext{
${ }^{1}$ The antipode acts on Lie algebra elements by multiplying them by -1 and thus acts on group elements by sending them to their inverse.
} 


\section{Lax pair and $r$-matrix for affine Toda theory}

The Lax pair for the Toda theory based on an untwisted ${ }^{2}$ affine Lie algebra $g^{(1)}$ takes values in the finite dimensional Lie algebra $g$, 17]

$$
\begin{aligned}
& a_{t}(\lambda)=\frac{1}{2} \partial_{x} \boldsymbol{\phi} \cdot \mathbf{H}+\sum_{i=0}^{n} \sqrt{\eta_{i} \alpha_{i}^{2} / 8}\left(\lambda E_{\alpha_{i}}-1 / \lambda E_{-\alpha_{i}}\right) e^{\alpha_{i} \cdot \boldsymbol{\phi} / 2} \\
& a_{x}(\lambda)=\frac{1}{2} \partial_{t} \boldsymbol{\phi} \cdot \mathbf{H}+\sum_{i=0}^{n} \sqrt{\eta_{i} \alpha_{i}^{2} / 8}\left(\lambda E_{\alpha_{i}}+1 / \lambda E_{-\alpha_{i}}\right) e^{\alpha_{i} \cdot \boldsymbol{\phi} / 2} .
\end{aligned}
$$

Substituting the expressions (3.1) into the zero curvature condition (2.1) and using the Lie bracket relations

$$
\left[\mathbf{H}, E_{\alpha}\right]=\boldsymbol{\alpha} E_{\alpha}, \quad\left[E_{\alpha}, E_{\alpha}\right]=0, \quad\left[E_{\alpha}, E_{-\alpha}\right]=\frac{2}{\alpha^{2}} \boldsymbol{\alpha} \cdot \mathbf{H} .
$$

reproduces the equations of motion (1.1).

The canonical equal-time Poisson bracket relations

$$
\left\{\phi_{a}(x, t), \partial_{t} \phi_{b}(y, t)\right\}=\delta_{a b} \delta(x-y)
$$

lead to Poisson brackets for the $a_{x}$ of the form (2.2) with the $r$-matrix [17]

$$
\begin{aligned}
r(\lambda) \propto \frac{\lambda^{h}+1}{\lambda^{h}-1} \sum_{a=1}^{n} H_{a} \otimes H_{a} & -\frac{2}{1-\lambda^{h}} \sum_{\alpha>0} \frac{\alpha^{2}}{2} \lambda^{l(\alpha)} E_{\alpha} \otimes E_{-\alpha} \\
& +\frac{2}{1-\lambda^{-h}} \sum_{\alpha>0} \frac{\alpha^{2}}{2} \lambda^{-l(\alpha)} E_{-\alpha} \otimes E_{\alpha}
\end{aligned}
$$

where the generators are chosen so that $\operatorname{tr}\left(H_{a} H_{b}\right)=\delta_{a b}$ and $\operatorname{tr}\left(E_{\alpha} E_{-\beta}\right)=2 \delta_{\alpha \beta} / \alpha^{2}$.

\section{Durham boundary conditions}

We can obtain the Durham boundary conditions (1.2) from the Sklyanin formalism by choosing the anti-automorphism $\sigma$ as transposition, acting on the Lie algebra generators as $E_{\alpha}^{t}=E_{-\alpha}$ and $H^{t}=H$. The condition (2.13) for $K_{+}$becomes

$$
\left[K_{+}(\lambda), \frac{1}{2} \partial_{x} \boldsymbol{\phi} \cdot \mathbf{H}\right]_{+}+\left[K_{+}(\lambda), \sum_{i=0}^{n} \sqrt{\eta_{i} \alpha_{i}^{2} / 8}\left(\lambda E_{\alpha_{i}}-1 / \lambda E_{-\alpha_{i}}\right) e^{\alpha_{i} \cdot \boldsymbol{\phi} / 2}\right]_{-}=0
$$

at $x=x_{+}$. This is exactly the same as equation (3.14) in $\mathbb{1}$ if one makes the identification $K_{+}=\mathcal{K}^{-1}$ and uses that $\partial B / \partial \phi=-\partial_{x} \phi$. In [1] this equation was derived by a different formalism in which the Lax pair condition gives both the

\footnotetext{
${ }^{2} \mathrm{~A}$ restriction which we make only for compactness of presentation
} 
equations of motion and the boundary conditions. The results of the Durham group show that there exists a $K_{+}(\lambda)$ satisfying eq.(4.1) only if the boundary condition is of the form eq.(1.2) with very restricted choice for the coefficients $A_{i}$ and that such a $K_{+}(\lambda)$ automatically satisfies the reflection equation (2.7) (Equation (4.8) in [1] is equivalent to our eq.(2.7) because the Toda $r$-matrix has the property that $\left.r^{t_{1} t_{2}}(\lambda)=-r(1 / \lambda)\right)$. The simplest solution is of course $K_{+}(\lambda)=1$ which solves (4.1) when the boundary condition is $\partial_{x} \phi\left(x_{+}\right)=0$.

If $K_{+}(\lambda)$ is a solution of eq.(2.13) then $K_{-}(\lambda)=K_{+}(-1 / \lambda)$ is a solution of eq. 2.14) with the same boundary condition. This is so because $a_{t}^{t}(-\lambda)=a_{t}(\lambda)$. This $K_{-}(\lambda)$ satisfies the reflection equation (2.8) because $r^{t_{1} t_{2}}(\lambda)=-r(1 / \lambda)$. Thus affine Toda theory is integrable on any interval $\left[x_{-}, x_{+}\right]$with boundary conditions of the form (1.2) at both ends, where the coefficients $A_{i}$ do not have to be the same at the two ends (but of course independently have to obey the restrictions of [1]). The Durham group dealt only with the half line which can be obtained by choosing $K_{-}=1$ and taking the limit $x_{-} \rightarrow-\infty$.

\section{The soliton-preserving boundary conditions}

We will now choose the anti-automorphism $\sigma$ to be hermitian conjugation $\dagger$, defined by its action on the Lie algebra generators $E_{\alpha}^{\dagger}=E_{-\alpha}$, and $H_{a}^{\dagger}=H_{a}$. It differs from transposition only in that any coefficients of Lie algebra generators will be complex conjugated and thus in particular $(\boldsymbol{\phi} \cdot \mathbf{H})^{\dagger}=\boldsymbol{\phi}^{*} \cdot \mathbf{H}$. The condition (2.13) of compatibility of the $K$-matrix with the Lax connection therefore reads

$$
\begin{aligned}
& 0=\left[K_{+}(\lambda), \frac{1}{2} \partial_{x} \operatorname{Re}(\phi) \cdot H\right]_{+}+\left[K_{+}(\lambda), \sum_{i=0}^{n} \sqrt{\frac{\eta_{i} \alpha_{i}^{2}}{8}}\left(\lambda E_{\alpha_{i}}-\frac{1}{\lambda} E_{-\alpha_{i}}\right) \operatorname{Re}\left(e^{\alpha_{i} \cdot \boldsymbol{\phi} / 2}\right)\right]_{-} \\
& 0=\left[K_{+}(\lambda), \frac{1}{2} \partial_{x} \operatorname{Im}(\boldsymbol{\phi}) \cdot H\right]_{-}+\left[K_{+}(\lambda), \sum_{i=0}^{n} \sqrt{\frac{\eta_{i} \alpha_{i}^{2}}{8}}\left(\lambda E_{\alpha_{i}}-\frac{1}{\lambda} E_{-\alpha_{i}}\right) \operatorname{Im}\left(e^{\alpha_{i} \cdot \boldsymbol{\phi} / 2}\right)\right]_{+}
\end{aligned}
$$

at $x=x_{+}$. If one imposes our new boundary condition (1.5) then these equations are solved by $K_{+}=1$ which is also a solution of the reflection equation (2.7). This proves integrability of the condition (1.5).

Also the $K$-matrices found by the Durham group can solve these equations but only if the boundary conditions are stronger than the conditions (1.2) which were already known to be integrable.

\section{Discussion}

We have seen that the Sklyanin formalism can be generalized by replacing the an- 
tipode by an arbitrary linear or antilinear antiautomorphism. By choosing this antiautomorphism to be transposition and using the Durham K-matrices [1] one obtains the known boundary conditions (1.2). By choosing hermitian conjugation instead and choosing $K=1$ one obtains the new boundary condition (1.5).

We did not explicitly extract local integrals of motion in involution from the generating functional $\tau(\lambda)$. This might perhaps be done by extending the abelianization formalism of Olive and Turok [17] to the situation with boundaries. Intuitively we expect the charges carried by the solitons to take the same values as on the whole line, determined by Freeman [9].

We are not claiming to have found all integrable boundary conditions. For example it is probably possible to find more integrable boundary conditions involving the time derivative of the field by using gauge transformed Lax pairs as in [2].

Gandenberger [11] has successfully determined the soliton-conjugating quantum reflection matrices for the Durham boundary conditions (1.2) for $g=\operatorname{sl}(3)$. We expect that it will be similarly possible to find the soliton-preserving quantum reflection matrices for our boundary condition (1.5) from the solutions of the reflection equation given in [8].

\section{Acknowledgments}

I would like to thank Ed Corrigan, Rafael Nepomechie and Petr Kulish for discussions during the Euroconference on "New Symmetries in Statistical Mechanics and Condensed Matter Physics" funded by TMR contract number ERBFMMACT970283. This work was supported in part by the European Commission TMR Network, contract number FMRX-CT96-0012. I thank the EPSRC for an advanced research fellowship.

\section{References}

[1] P. Bowcock, E. Corrigan, P.E. Dorey, R.H. Rietdijk, Classically integrable boundary conditions for affine Toda field theories, Nucl. Phys. B 445 (1995) 469, hep-th/9501098.

[2] P. Bowcock, E. Corrigan, R. H. Rietdijk, Background field boundary conditions for affine Toda field theories, Nucl. Phys. B 465 (1996) 350-364, hep-th/9510071.

[3] Y.-X. Chen, X.-D. Luo and K. Wu, Classically and Quantum Integrable Systems with Boundary, hep-th/9808150.

[4] E. Corrigan, P.E. Dorey, R.H. Rietdijk and R. Sasaki, Affine Toda field theory on a half line, Phys. Lett. B 333 (1994) 83, hep-th/9404108.

[5] E. Corrigan, Recent developments in affine Toda field theory, hep-th/9412213. 
[6] E. Corrigan, P.E. Dorey and R.H. Rietdijk, Aspects of affine Toda field theory on a half line, Prog.Theor.Phys.Suppl. 118 (1995) 143-164, hep-th/9407148.

[7] G.W. Delius, Restricting affine Toda theory to the half-line, hep-th/9807189.

[8] H.J. de Vega and A. González-Ruiz, Mod. Phys. Lett. A9 (1994) 2207.

[9] M. Freeman, Conserved charges and soliton solutions in affine Toda theory, Nucl. Phys. B433 (1995) 657-670, hep-th/9408092.

[10] G.M. Gandenberger, Exact S-matrices for Quantum Affine Toda Solitons and their Bound States, Ph.D. thesis, University of Cambridge 1996, available as postscript file at http://www.damtp.cam.ac.uk/user/hep/publications.html

[11] G.M. Gandenberger, On $a_{2}^{(1)}$ Reflection Matrices and Affine Toda Theories, hep-th/9806003.

[12] S. Ghoshal and A.B. Zamolodchikov, Boundary $S$ matrix and boundary state in two-dimensional integrable field theory, Int. J. Mod. Phys. A 9 (1994) 3841, hep-th/9306002.

[13] T.J. Hollowood, Quantum Solitons in Affine Toda Field Theories, Nucl. Phys. B 384 (1992) 523, hep-th/9110010.

[14] T. J. Hollowood, Quantizing SL(N) Solitons and the Hecke Algebra Int. J. Mod. Phys. A 8 (1993) 947-982, hep-th/9203076.

[15] P. P. Kulish, R. Sasaki and C. Schwiebert, Constant Solutions of Reflection Equations and Quantum Groups, J. Math. Phys. 34 (1993) 286-304, hep-th/9205039.

[16] A. MacIntyre, Integrable boundary conditions for classical sine-Gordon theory, hep-th/9410026.

[17] D.I. Olive and N. Turok, Local conserved densities and zero-curvature conditions for Toda lattice field theories, Nucl. Phys. B 257 (1985) 277-301.

[18] D.I. Olive, N. Turok and J.W.R. Underwood, Solitons and the energy-momentum tensor for affine Toda theory, Nucl. Phys. B 401 (1993) 663, Affine Toda Solitons and Vertex Operators Nucl. Phys. B 409 (1993) 509, hep-th/9305160.

[19] H. Saleur, S. Skorik and N.P. Warner, The boundary sine-Gordon theory: classical and semi-classical analysis, Nucl. Phys. B 441 (1995) 421-436, hep-th/9408004.

[20] E.K. Sklyanin, Boundary conditions for integrable equations, Funct. Anal. Appl. 21 (1987) 164. 